\title{
Structural and Genetic Diversity of Entamoeba Gingivalis Trophozoites Isolated From Diseased and Healthy Periodontal Sites
}

Marwa A. EL-Dardiry ( $\square$ marwaahmed2008@outlook.com )

Fayoum University

Amany A. Abdel-Aal

Cairo University

Reham K. Nahnoush

Cairo University

Marwa A. Elmallawany

Cairo University

Mohamed Akmal

Cairo University

Adam A. Afife

Leicester University

Mohamed S. Badr

Ain Shams University

\section{Research Article}

Keywords: Entamoeba gingivalis, genotypes, real-time PCR, HRM, mutants.

Posted Date: August 5th, 2021

DOI: https://doi.org/10.21203/rs.3.rs-774043/v1

License: (c) (i) This work is licensed under a Creative Commons Attribution 4.0 International License. Read Full License 


\section{Abstract}

The variability aspects of Entamoeba gingivalis concerning periodontal diseases are poorly studied. The reason is that many researchers rejected the notion that it can cause periodontal disease. The current study aimed to compare the morphological and genetic variability within trophozoites isolated from diseased and healthy periodontal sites. The practical investigation included a detailed microscopic analysis and gene scanning of the 18S-SSU rRNA by real-time PCR. All trophozoites isolated from diseased sites were significantly larger than those from healthy sites. Besides, they were clustered together with many other leuko-phagocytes. Gene scanning revealed diversity within the isolates of mutant trophozoites isolated from diseased sites. Four melting curves matched the $\mathrm{H} 57$ strain, while the remaining eight expressed the wild strain. Most of the isolates from healthy sites were wild-type, while only two isolates represented the H57 strain. However, the study confirmed morphological and genetic variability between different isolates, more in-depth molecular studies are required to investigate the role of this oral protozoan in the pathogenicity of periodontal affection. The study highlighted the importance of multidisciplinary diagnostic strategies by employing exported clinical investigators to reach truthful scientific outcomes concerning the association of certain microorganisms to particular diseases or disorders.

\section{Introduction}

Entamoeba gingivalis is a protozoan that usually exists in the oral cavity in trophozoite form without a cyst stage, thus requiring a specific contact via different means to be transmitted. Generally, all scientific studies concerning variable aspects related to this oral protozoan are limited and controversial. That is probably due to the denial by some clinical specialists that it may cause such oral diseases. The difficulties facing the direct diagnosis of this oral parasite and the complexity in maintaining the parasite in vitro added a burden to the researchers in this field which certainly reflected the number of published researches concerning this infection (Ghabanchi et al., 2010 and Cembranelli et al., 2013). several studies considered E. gingivalis a commensal organism that is found commonly within bacterial plaques and saliva (Wantland and Lauer, 1970; Favoreto Junior and Machado, 1995 and Gharavi et al., 2006). Again, its pathogenicity is subject to debate because the protozoan had been identified in both healthy individuals as well as patients suffering from periodontal disease, either immunocompetent or immunocompromised (Trim et al., 2011).

Kikuta and his colleagues (1996) were the first research team to successfully amplify the small subunit of ribosomal RNA gene (SSU rDNA), using laboratory cultured E. gingivalis and designing their original specific primers. Subsequently, these specific primers were used to amplify the DNA isolated from dental plaques of patients suffering from periodontal disease by performing both conventional and real-time quantitative PCR (qPCR). Surprisingly, the authors recorded a much higher sensitivity of the qPCR than the conventional PCR as it failed to detect $42 \%$ of cases proven to be positive by both microscopy and qPCR. These shocking facts about conventional PCR limit its usage as an independent technique in scientific research. 
Unfortunately, the genetic diversity of such oral parasites and their role in the prognosis of periodontal disease or the damaging consequences is not well studied. Cembranelli et al. (2013) recorded the first evidence of E. gingivalis genetic variability and its unhealthy oral manifestations among HIV/AIDS immunocompromised patients by using the applications of conventional PCR and Sanger sequencing. In (2018), Garcia et al. discovered a high genetic diversity between 2 different $E$. gingivalis isolates, identified in different categories of human subjects. The authors named the strain isolated from the infected patients as ST2 kamaktli variant and documented its prominent genetic deviation as well as its virulence from the ST1 E. gingivalis strain, performing the same consecutive molecular techniques; PCR followed by sequencing. However, these authors did not relate the identified variants with any morphological features.

In a study from the University of Utah by Idaho Technology, a high-resolution melting curve (HRM) analysis was innovated to minimize the cost of the molecular techniques, besides, investigating the genetic diversity in a particular organism (Garritano et al., 2009). This technique facilitates the rapid, sensitive, and easy detection of genetic polymorphisms and mutations by real-time PCR analysis and further increased the ability to detect the epigenetic variations within the double-stranded DNA. The technique necessitates a real-time PCR machine with exceptional thermal stability and sensitivity, plus a specific software analytic program (Garritano et al., 2009).

The current study assessed the possibility of E. gingivalis genetic variability with morphological characters within healthy subjects versus patients suffering from periodontal diseases, performing HRM technology following real-time amplification of the lower ribosomal subunit of the $18 \mathrm{~S}$ region (18S-SSU rRNA).

\section{Results}

\section{Morphological characteristics of E. gingivalis:}

The current study involved 40 samples with positive E. gingivalis infection which were confirmed microscopically and molecularly by real-time PCR. These 40 isolates were obtained from 2 categories of subjects; group A; 26 patients suffering from chronic periodontal diseases (14 males and 12 females with mean age, $32.923 \pm 5.214$ years), plus 14 healthy volunteers (eight males and six females; with mean age, $33.429 \pm 4.484$ years). Noticeably, these 40 positive samples were identified and enrolled in the study after examining samples from a total of 144 subjects. Regarding the parasitological confirmation, motile E. gingivalis trophozoites were evident microscopically within all 40 positive specimens. The trophozoites were smaller than that of Entamoeba histolytic. They were observed with the characteristic morphological features in the form of a single nucleus containing a tiny central karyosome with a rim of chromatin at the periphery and a delicately granular cytoplasm (Fig. 1).

It was noted that the inclusion bodies in the form of leukophagocytes were found within the cytoplasm of all samples taken from diseased cases. In addition, numerous trophozoites were found in bunches; 
resembling nests, within most of the samples isolated from the diseased group (17 out of the 26). Fewer numbers of trophozoites were noticed in samples taken from healthy subjects. Interestingly, the size of trophozoites isolated from diseased cases $(18.554 \pm 1.375 \mu$ ) was significantly larger than of those taken from healthy subjects $(12.679 \pm 0.944 \mu)$. Significantly more trophozoites were identified in patients with periodontal disease compared to healthy patients. The tested samples from patients showed $86.577 \pm$ 11.04 of trophozoites, in ten microscopic fields, compared to $5.357 \pm 1.905$ of trophozoites in samples from healthy subjects.

\section{Genetic diversity of different strains of E. gingivalis in both of healthy and diseased sites:}

Gene scanning revealed diversity within the isolates with a significantly higher number of mutants (18 out of 26) within the trophozoites isolated from diseased sites, 14 of them were of unknown origin. Four melting curves matched $E$. gingivalis $\mathrm{H} 57$ strain, while the remaining eight curves were related to the wild strain (ATCC-30927). 12 out of the 14 isolates from healthy sites corresponded to the wild type while only two of them related to the $\mathrm{H} 57$ strain (Table 1, Fig. 2). 
Table 1

Demographic data, oral health condition, morphological features and genetic types of E. gingivalis related to diseased and healthy subjects.

\begin{tabular}{|c|c|c|c|c|c|}
\hline \multicolumn{3}{|l|}{ Variables } & $\begin{array}{l}\text { Diseased } \\
\text { subjects } \\
(n=26)\end{array}$ & $\begin{array}{l}\text { Healthy } \\
\text { subjects } \\
(n=14)\end{array}$ & $\begin{array}{l}P \text { - } \\
\text { value }\end{array}$ \\
\hline \multirow[t]{3}{*}{ Demographic data } & \multicolumn{2}{|c|}{ Age (years) } & $32.923 \pm 5.214$ & $\begin{array}{l}33.429 \pm \\
4.484\end{array}$ & 0.598 \\
\hline & \multirow[t]{2}{*}{ Sex } & Male $(n=22)$ & $14(53.8 \%)$ & $8(57.1 \%)$ & \multirow[t]{2}{*}{0.842} \\
\hline & & Female $(n=18)$ & $12(46.2 \%)$ & $6(42.9 \%)$ & \\
\hline \multirow[t]{2}{*}{ Oral hygiene } & \multicolumn{2}{|c|}{ Poor $(n=27)$} & $23(88.5 \%)$ & $4(28.6 \%)$ & \multirow[t]{2}{*}{$0.000 *$} \\
\hline & \multicolumn{2}{|c|}{$\operatorname{Good}(n=13)$} & $3(11.5 \%)$ & $10(71.4 \%)$ & \\
\hline $\begin{array}{l}\text { Morphological } \\
\text { features of }\end{array}$ & \multicolumn{2}{|c|}{$\begin{array}{l}\text { Number of trophozoites/10 } \\
\text { field }\end{array}$} & $86.577 \pm 11.04$ & $5.357 \pm 1.905$ & $0.000 *$ \\
\hline \multirow[t]{3}{*}{ E. gingivalis } & \multicolumn{2}{|c|}{ Size of trophozoites } & $18.554 \pm 1.375 \mu$ & $\begin{array}{l}12.679 \pm \\
0.944 \mu\end{array}$ & $0.000 *$ \\
\hline & \multicolumn{2}{|c|}{ Leukophagocytes } & $26(100 \%)$ & $5(35.7 \%)$ & $0.000 *$ \\
\hline & \multicolumn{2}{|c|}{ Bunches of trophozoites } & $17(65.4 \%)$ & $0(0 \%)$ & $0.000 *$ \\
\hline \multirow[t]{4}{*}{ E. gingivalis strains } & \multicolumn{2}{|c|}{ Wild type } & $8(30.8 \%)$ & $12(85.7 \%)$ & \multirow[t]{4}{*}{$0.000 *$} \\
\hline & \multicolumn{2}{|l|}{$\mathrm{H} 57$} & $4(15.4 \%)$ & $2(14.3 \%)$ & \\
\hline & \multicolumn{2}{|l|}{ C } & $0(0 \%)$ & $0(0 \%)$ & \\
\hline & \multicolumn{2}{|c|}{ Mutant } & $14(53.8 \%)$ & $0(0 \%)$ & \\
\hline
\end{tabular}

* Statistically significant, $p$-value $<0.05$

\section{Discussion}

The world health organization (WHO) considered periodontitis as a handicapping disease due to its damaging consequences affecting a population's health (WHO, 2016). Entamoeba gingivalis parasitic trophozoites were frequently detected in oral cavities, yet the association of periodontitis with this protozoan is still debatable, fluctuating from complete accusation to intense rejection of such an association (Bonner et al., 2018). Very few published scientific studies relied on the specific parasitological parameters to identify the oral protozoan microscopically, yet nearly all of them confirmed its $100 \%$ occurrence within progressive periodontal pockets and from 0 to $26 \%$ within healthy periodontal sites (Fisher, 1927; Hinshaw and Simonton, 1928; Wantland and Wantland, 1960; Wantland and Lauer, 1970; Gottlieb and Miller, 1971; Keyes and Rams, 1983; Lange, 1984; Linke et al., 1989 and Hassan et al., 2019). Unfortunately, for unknown reasons, all these articles neglected the difference in morphological 
characteristics between the detected trophozoites among the diseased and healthy periodontal sites, except Hassan et al. (2019) in which detailed information was reported, yet not specifically with the different conditions. That is why this study aimed to investigate this structural issue in addition to the molecular investigation proposed to detect genetic variability within the isolates.

The current study revealed significant morphological differences between E. gingivalis trophozoites within diseased and healthy sites including the size of parasitic stages, specific morphological variations in addition to the mean number of trophozoites between the 2 categories. Detailed morphological features reported in this study were sufficient to differentiate the parasitic stages within the diseased and healthy periodontal sites. This was in contrast to the findings reported by García et al. (2018) in which morphological characteristics were ignored and excluded by the authors. They attributed this to the ineffectuality of microscopic examination to differentiate between amoebas. Similarly, other authors reported similar findings concerning morphology, in which no characteristic findings were reported (Clark et al. 2006; Jacob et al. 2016; Ponce-Gordo and Martinez-Diaz 2010; Stensvold et al. 2011; Weedall and Hall 2011).

García et al. (2018) highlighted an example for such a problem, however, it was related to E. histolytica and E. dispa. They stated that both amoebas are morphologically similar, but their genetic and pathogenic differences allow them to be classified as independent, although closely related species (Clark 2000). However, many authors recorded a clear microscopic difference related to erythrophagocytic activity which was observed in the former species but never observed in the latter one.

These unique microscopic findings are certainly in need of professional specialists, not to be misdiagnosed or misinterpreted. Thereby supporting the urgent need for real engagement of multidisciplinary diagnostic strategies, involving specialists from a variety of medical fields to reach truthful scientific outcomes concerning the association of certain microorganisms with particular diseases or disorders.

Morphologically in this study, in addition to the observed characteristic nuclei, leuckophagocytic vacuoles were found within all the trophozoites isolated from affected periodontal sites. This may be related to certain virulence factors, facilitating the invasive power of E. gingivalis trophozoites, which is more or less similar to what is reported by Bansal et al. (2009), but in connection with erythrophagocytic ability of Entamoeba histolytica. This ability was not documented within the non-pathogenic E. dispar which has morphological features similar to E. histolytica. Phagocytosis of blood elements has been proposed as a pathogenicity indicator as well as a prominent virulence marker for Entamoeba species (Trissl et al., 1978 and Bhattacharya et al., 2002).

The significant difference regarding the larger size of E. gingivalis trophozoites identified within the diseased sites, possibly resulting from engulfing several WBCs which may have cause cytoplasmic expansion. E. gingivalis can engulf one or more human cells at a time, mainly polymorphonuclear cells and neutrophils. These are the chief cells within the periodontal pockets, so engulfing these cells may expose the first line of the innate immune mechanism to intense danger by consuming its powerful 
weapon (Bonner et al., 2014). Thus, E. gingivalis finds a perfect environment involving damaged tissue, bacteria, and fungi, permitting its safe embellishment and colonization. This may explain the significantly higher number within samples isolated from the diseased cases, taking into consideration that all the enrolled subjects were immunocompetent. This intense colonization within immunocompetent subjects was previously reported in other work published by Hassan et al. (2019).

Garcia et al. (2018) suggested the existence of different parasitic subtypes with the higher proliferative ability and/or proteolytic activity. This explains why this study intended to molecularly scan the different isolates, performing post-real-time PCR HRM to detect possible genetic variability between them. In this study, a significantly higher number of wild-type trophozoites was reported within healthy subjects. While a higher number of mutants was reported within diseased cases which were related to the known H57 strain of Cembranelli et al. (2013), plus unknown mutants revealed by HRM analysis (14 out of 26). Mutations observed in this work may have been triggered as a result of changes within the oral environment, which possibly turn the commensal amoebae into a pathogenic one, creating a vicious circle that facilitates more periodontal damage without cellular immune controlling mechanism, resulting in acceleration of amoebic colonization and so on. This is in concordance with the report of Tonetti et al. (2018) who documented the presence of E. gingivalis in some sulci that were not causing any symptoms as well as in diseased sites. However, certain conditions that change the surrounding environment can cause pathogenic conditions in the oral cavity.

In general, molecular tools such as specific target gene amplification and sequencing certainly expand genetic information concerning E. gingivalis. The work of Yamamoto et al. (1995) succeeded to molecularly identify this oral protozoan, using a long amplicon of $1400 \mathrm{bp}$. Their work has invoked more debate concerning the molecular identity of the parasite documented by various investigators. Kikuta et al. (1996) and Trim et al. (2011) molecularly detected the parasite only in diseased periodontal sites. While Bonner et al. (2014) and Deng et al., (2017) molecularly identified the parasite within $33.3 \%$ and $60 \%$ of healthy subjects respectively. The authors explained their higher molecular results within the healthy subjects to be due to their accurate control of PCR inhibitors and matrix degradation.

Genetic variability of $E$. gingivalis has been recognized by Cembranelli et al. (2013) and Garcia et al. (2018b) by studying the genetic variability of 18S-SSU rDNA. Furthermore, genetically identical $E$. gingivalis species revealed different virulence factors as reflected by the transcriptomic study (SantiRocca et al., 2008). This may explain the discrepancies in their molecular detection when compared with microscopy or clinical diagnosis as reported in this study. García et al. (2018) documented the presence of a kamaktli E. gingivalis variant which is located within a closely related clade to ST1, but the authors confirmed its mutability and named it as ST2. However, they did not mention any link to pathogenesis or anything related to the periodontal manifestation. Formerly, Clark and Diamond (1997) characterized three samples of $E$. gingivalis by riboprinting the $18 \mathrm{~S}$ rRNA region. Differential banding patterns observed after sample treatment with the restriction enzyme Rsa1 allowed them to categorize these 3 samples into two ribodemes: ribodeme-1 (two oral isolates) and ribodeme- 2 (one uterine isolate). 
Dissimilar band patterns created by kamaktli variants (Garcia and his co-workers, 2018) suggested its disparity from both ribodemal strains 1 and 2. The authors suggested that the unidentified E. gingivalis genetic type described by Cembranelli et al. (2013) in immunocompromised patients is possibly related to the kamaktli variant.

On the other hand, no cyst stage was found within our samples and all the observed parasitic stages were related to the E. gingivalis trophozoite stage. This was in concordance with most of the aforementioned documented reports. The report of García et al. (2018) triggered a new debate about E. gingivalis when they suggested a possible ability of their kamaktli variant to produce a cyst stage which had never been suggested before, still leaving the morphology of such a variant in the pending situation. Before this, Stensvold et al. (2011) advised the investigators in this field to postpone any formal taxonomic categorization until clear morphological data are obtainable. However, the kamaktli variant was categorized by the authors without any identifiable morphological characteristics, even the parasitic stage's level. Trim et al. (2011) discussed this issue and concluded that; E. histolytica is the only welldocumented human pathogen among all known amoebae and recommended the urgent need of an accurate and efficient detection methodology to correctly document other types of amoebae. Unfortunately, the debate concerning the role or the ability of some amoebae to initiate pathogenic effects within certain tissues (as in E. gingivalis) is still considered as a big obstacle facing the deeper understanding of its role in the pathogenicity of periodontal infection.

In this study, samples were collected from at least three different sites in each subject and confirmed positive after repeated microscopic examination by specialists. Only samples with positive microscopic confirmation were included, otherwise, all samples proven to be negative were excluded. This may be due to the nature of our study which was not intentionally designed to compare variable diagnostic tools. The design was mainly targeting the detection of morphological and genetic diversity within different isolates. Therefore, parasitological confirmation, involving detailed morphological features was a must. This possibly facilities specific figuring of isolates from different subject categories and possibly supported distinguishing each of them. Real-time PCR is an extremely sensitive molecular technique that operates within a closed system, thus avoiding the possible contamination during the post-polymerization process in conventional PCR. EvaGreen dye was used in our technique which is a third-generation dye developed to avoid PCR inhibition which may occur during the use of other dyes (Martino et al., 2010). In the current study, another benefit was achieved by this molecular technique which was the facilitation of simultaneous discovery of mutations using HRM software analysis and synthetic reference strains. Together, parasitological and molecular results supported the concept that E. gingivalis is one of the contributors to periodontal diseases, hence necessitating proper management to avoid the unnecessary destructive impact of this oral protozoal infection.

In conclusion, this study confirmed the morphological and genetic variability between different isolates and recommends further in-depth molecular studies to investigate the role of this oral protozoan in the pathogenicity of periodontal affection. This study also highlighted the importance of real engagement of multidisciplinary diagnostic strategies, involving experts from a variety of medical fields to reach truthful 
scientific outcomes concerning the association of certain microorganisms with particular diseases or disorders.

\section{Subjects, Patients And Methods}

\section{Study design and sample collection:}

The present cross-sectional study was approved by the ethical committee of the Faculty of Medicine, Cairo University, following the guidelines of the Council for International Organizations of Medical Sciences (CIOMS) available from: https://cioms.ch/wp-content/uploads/2017/01/WEB-CIOMSEthicalGuidelines.pdf. All consented forms were approved, signed, and collected from all patricians following the Declaration of Helsinki guidelines; available from: https://www.wma.net/policies-post/wmadeclaration-of-helsinki-ethical-principles-for-medical-research-involving-human-subjects/.

Subjects attending outpatient clinics of oral medicine at the Faculty of Dentistry, Cairo University, and suffering from periodontal diseases were enrolled in this study and informed consent was obtained from all enrolled subjects. Patients who recently received antibiotics or periodontal therapy, three months before enrollment, pregnant and immune-compromised subjects were all excluded from the study. A full oral clinical examination was performed at 6 locations, involving all teeth and considering the specific periodontal parameters according to Löe (1969); gingival index, plaque index, probing depth in addition to clinical attachment level. The diseased cases were managed and followed up according to the current institutional protocol. Patients were subjected to a complete oral examination involving visibly carious, tooth number and degree of mobility, evident plaques, and gingival bleeding plus other features.

Sample collection was done ensuing cautious drying of the selected sites, using a periodontal probe for healthy controls. In patients suffering from periodontitis, a sterile curette was used at the affected locations, and samples were obtained from periodontal pockets greater than $4 \mathrm{~mm}$. Following this, the specific mechanical management was completed by the specialist. Samples from each subject were divided into 2. The first portion was diluted using Polyvinyl alcohol (PVA) solution at 25-28oC to be triple stained with Iron \& hematoxylin according to the manufacturing instruction's manual (I\&H, Dalynn Biologicals, catalog No.SI70\&71). Then parasitological analysis was performed, using high power and oil immersion magnifications. E. gingivalis trophozoites were identified by their characteristic features, relying on its unique nucleus, pseudopodia, vacuoles, and leucko-phagocytic inclusions (Ibrahim and Abbas, 2012). The size of the parasitic stages was microscopically measured, according to Bailey (2012). Observed trophozoites were measured using an ocular micrometer that was calibrated against the stage micrometer in combination with the specific objective lens. For molecular technique, $100 \mu \mathrm{l}$ of RNAlater TM QiagenR solution was added to the second portion of each sample, placed in a sterile vial, and kept at $-20^{\circ} \mathrm{C}$ until used for molecular analysis.

\section{DNA extraction and Amplification of E. gingivalis 18S- SSU rRNA by qPCR:}


Following the manufacturer's instructions, DNA from each sample was extracted using the QIAamp DNA Mini kit (Qiagen, Germany). The extracted DNA samples were quantified before molecular analysis using spectrophotometric analysis. The real-time PCR technique was performed qualitatively in this work intended for post-amplification HRM analysis, using the original primers designed by Kikuta et al. (1996) for samples deemed positive microscopically by parasitologists. The molecular technique was performed for post-amplification gene scanning, so no standard curve was created. The primers were purified by HPLC and purchased from (Metabion, Planegg, Germany). For amplification and HRM analysis, the LightCycler ${ }^{\circledR} 480$ operator's manual was followed with a ready-to-use $2 X$ concentration, hot start reaction mix designed for amplification and detection of specific DNA sequence in the presence of the proper PCR primers. This step was followed by HRM analysis to detect variants among different isolates, taking into consideration the easy optimization of $\mathrm{Mg} 2+$ concentration supplied with the system ( $\mathrm{MgCl} 2$ stock solution) to avoid nonspecific byproducts. According to the operator's manual, $10 \mu$ of the master mix, $2 x$ concentrated was used, $1.0 \mu \mathrm{l}$ for a final concentration, for each of the primers, $0.2 \mu \mathrm{m}$ was added in the PCR reaction; forward primer; (5'-GAATAGGCGCATTTCGAACAGG-3'), reverse primer; (5'-

TCCCACTAGTAAGGTACTTACTC - 3'), $5 \mu$ of the extracted genomic DNA and the reaction mixture was adjusted to a final volume of $20 \mu \mathrm{L}$ using PCR grade water. The whole procedure was done in 75 minutes including 10 minutes pre-incubation and 15 minutes HRM. The condition parameters were as follows; one cycle pre-incubation, hot start $95^{\circ} \mathrm{C}$ for $7.5 \mathrm{~min}, 45$ cycle amplification of $95^{\circ} \mathrm{C}$ for one minute, followed by touchdown protocol covering a range of annealing temperatures from $65^{\circ} \mathrm{C}$ to $53^{\circ} \mathrm{C}$, then $72^{\circ} \mathrm{C}$ for $30 \mathrm{~s}$, followed by a 5 -min extension at $72^{\circ} \mathrm{C}$. All samples were run in duplicate and negative control was included by replacing the template DNA with water PCR Grade supplied within the kit.

\section{Gene scanning and high-resolution melting curve analysis}

According to Martino et al. (2010), the analysis was done using hot start technology and EvaGreen dye. Samples with variations in DNA sequence were differentiated by discrepancies in melting curve shape, compared to reference synthetic genes. The process began at $95^{\circ} \mathrm{C}$ for $5 \mathrm{~min}$ followed by a $0.5^{\circ} \mathrm{C}$ decrease in temperature every $30 \mathrm{~s}$ to $47^{\circ} \mathrm{C}$.

\section{Internal control and reference genes}

Synthetic copies of the three sequences of E. gingivalis genes identified by Cembranelli et al. (2013) [Finan Company, Egypt] were used in the current study as reference genes, according to Abdilmawjood et al. (2002). Genetic sequences corresponding to these strains were obtained from GenBank and identified by their accession number. The original reference strain, ATCC-30927 (D28490), and the polymorphic E. gingivalis C (KF250433) and E. gingivalis H57 (KF250436) strains with single nucleotide polymorphism (SNP). For logical as well as financial reasons, there was no need to use the other two E. gingivalis strains (E. gingivalis $\mathrm{H} 14$ and $\mathrm{E}$ ), documented by the authors, as they had the same sequence of $\mathrm{H} 57$ strain. For the detection of E. gingivalis and its mutants, two types of original plasmid, "wild" (W) and "mutation" (M1 and M2) plasmids, were created using the TA cloning kit (Invitrogen Corp., San Diego, CA).

\section{Statistical analysis:}


The statistical analysis was performed by SPSS statistics software version 24 (IBM, NY, USA). Qualitative data were expressed as percentage and frequency. Numerical data were expressed as mean or range. The Chi-square test was used to determine the relationship between qualitative variables. Probability value ( $P$ value) $P<0.05$ indicated a statistically significant correlation while $P>0.05$ was insignificant.

\section{Declarations}

Availability of data and materials: The datasets used and/or analyzed during the current study are available from the corresponding author on reasonable request.

Competing interests: The authors declare that they have no competing interests.

Funding: This study is not funded.

Authors' contributions: M. A. Ed. and A. A. Aa. contributed to the conception and study design. M. S. B. and R. K. N. contributed to the editing and reviewing of the intellectual contents. M. A. Ed, M. A. E. and M. A. were responsible for the literature search besides the experimental and clinical applications A. A. Aa., A. A. A, and M. A. Ed. contributed to the data acquisition. A. A-A. and M. A. were responsible for the statistical analysis. M. A. Ed. and M.S.B. contributed to the data analysis, manuscript preparation, editing and reviewing. M. A. Ed. acts as a guarantor and corresponding author.

Acknowledgements: Not applicable.

\section{References}

1. Abdulmawjood, A., Roth, S. \& Bülte, M. Two methods for construction of internal amplification controls for the detection of Escherichia coli 0157 by polymerase chain reaction. Mol. Cell, Probes, 16, 335-339 (2002).

2. Bailey, V. L. et al. Measurements of microbial community activities in individual soil macroaggregates. Soil Biol. Biochem, 48, 192-195 (2012).

3. Bansal, D. et al. An ex-vivo human intestinal model to study Entamoeba histolytica pathogenesis. PLoS Negl. Trop. Dis, 3, e551 https://doi.org/10.1371/journal.pntd.0000551 (2009).

4. Bhattacharya, S., Bhattacharya, A. \& Petri, W. A. Jr. Examining Entamoeba. in Trends in Parasitology196-197(CelPress, 2002).

5. Bonner, M. et al. Detection of the amoeba Entamoeba gingivalis in periodontal pockets. Parasite, 21, 30 https://doi.org/10.1051/parasite/2014029 (2014).

6. Bonner, M., Fresno, M., Gironès, N., Guillén, N. \& Santi-Rocca, J. Reassessing the Role of Entamoeba gingivalis in Periodontitis. Frontiers in cellular and infection microbiology, 8, 379 https://doi.org/10.3389/fcimb.2018.00379 (2018).

7. Cembranelli, S. B. et al. First evidence of genetic intraspecific variability and occurrence of Entamoeba gingivalis in HIV(+)/AIDS. PloS one, 8, e82864 
https://doi.org/10.1371/journal.pone.0082864 (2013).

8. Clark, C. G. The evolution of Entamoeba, a cautionary tale. Res. Microbiol, 151, 599-603 (2000).

9. Clark, C. G. \& Diamond, L. S. Intraspecific variation and phylogenetic relationships in the genus Entamoeba as revealed by riboprinting. J. Eukaryot. Microbiol, 44, 142-154 (1997).

10. Clark, C. G. et al. New insights into the phylogeny of Entamoeba species provided by analyses of four new small-subunit rRNA genes. Int. J. Syst. Evol. Microbiol, 56, 2235-2239 (2006).

11. Trissl, D., Martinez-Palomo, A., de la Torre, M., de la Hoz, R. \& de Perez, E. Fagocitosis de eritrocitos humanos por Entamoeba histolytica. Estudio cuantitativo [Phagocytosis of human erythrocytes by Entamoeba histolytica. Quantitative study]. Arch. Invest. Med. (Mex), 9, 219-222 (1978).

12. Deng, Z. L., Szafrański, S. P., Jarek, M., Bhuju, S. \& Wagner-Döbler, I. Dysbiosis in chronic periodontitis: Key microbial players and interactions with the human host. Sci. Rep, 7, 3703 https://doi.org/10.1038/s41598-017-03804-8 (2017).

13. Fisher, J. H. Pyorrhea alveolaris: the rôle of certain microörganisms found in the lesions. Am. J. Pathol, 3, 169 (1927).

14. García, G. et al. A new subtype of Entamoeba gingivalis: "E. gingivalis ST2, kamaktli variant". Parasitol. Res, 117, 1277-1284 (2018).

15. Garritano, S., Gemignani, F., Voegele, C., Nguyen-Dumont, T. \& Calvez-Kelm, L. F., et al. Determining the effectiveness of High-Resolution Melting analysis for SNP genotyping and mutation scanning at the TP53 locus. BMC genetics, 10, 5 https://doi.org/10.1186/1471-2156-10-5 (2009).

16. Gottlieb, D. S. \& Miller, L. H. Entamoeba gingivalis in periodontal disease. J. Periodontol, 42, 412-415 (1971).

17. Hassan, S. S., Madkour, G. G., Henin, R. W. \& Gad, S. W. F. Abd El-Aal, A. A. Is Entamoeba Gingivalis a Risk Factor for Periodontal Diseases? A Case-Control Study. Perio J, 3, 18-28 (2019).

18. Hinshaw, H. C. \& Simonton, F. The amoebas of the mouth in relation to incidence of pyorrhea, clinical symptoms, extent of lesions, and age of patients. J. Dent. Res, 8, 507-528 (1928).

19. Ibrahim, S. \& Abbas, R. Evaluation of Entamoeba gingivalis and Trichomonas tenax in patients with periodontitis and gingivitis and its correlation with some risk factors. J. Baghdad Coll. Dent, 24, 158162 (2012).

20. Jacob, A. S., Busby, E. J., Levy, A. D., Komm, N. \& Clark, C. G. Expanding the Entamoeba Universe: New Hosts Yield Novel Ribosomal Lineages. J. Eukaryot. Microbiol, 63, 69-78 (2016).

21. Keyes, P. H. \& Rams, T. E. A rationale for management of periodontal diseases: rapid identification of microbial 'therapeutic targets' with phase-contrast microscopy. J. Am. Dent. Assoc, 106, 803-812 (1983).

22. Kikuta, N., Yamamoto, A. \& Goto, N. Detection and identification of Entamoeba gingivalis by specific amplification of rRNA gene. Can. J. Microbiol, 42, 1248-1251 (1996).

23. Lange, D. E. Microbiological aspects of periodontal diseases. Zahnarztl. Mitt, 74, 2420242224202423 (1984). 
24. Linke, H. A., Gannon, J. T. \& Obin, J. N. Clinical survey of Entamoeba gingivalis by multiple sampling in patients with advanced periodontal disease. Int. J. Parasitol, 19, 803-808 (1989).

25. Löe, H. The Gingival Index, the Plaque Index and the Retention Index Systems. J. Periodontol, 38, 610-616 (1967).

26. Martino, A., Mancuso, T. \& Rossi, A. M. Application of high-resolution melting to large-scale, highthroughput SNP genotyping: a comparison with the TaqMan method. J. Biomol. Screen, 15, 623-629 (2010).

27. World health organization. Global Health Estimates 2015: Deaths by Cause, Age, Sex, by Country and by Region, 2000-2015. Geneva. (2016).

28. Ponce-Gordo, F. \& Martinez-Diaz, R. A. Taxonomía y filogenia del género Entamoeba. Una revision histórica [Taxonomy and phylogeny of Entamoeba species. An historical review]. Rev. IberoLatinoam. de Parasitol, 69, 5-37 (2010).

29. Santi-Rocca, J. et al. The lysine- and glutamic acid-rich protein KERP1 plays a role in Entamoeba histolytica liver abscess pathogenesis. Cell Microbiol, 10, 202-217 (2008).

30. Stensvold, C. R. et al. Increased sampling reveals novel lineages of Entamoeba: consequences of genetic diversity and host specificity for taxonomy and molecular detection. Protist, 162, 525-541 (2011).

31. Tonetti, M. S., Greenwell, H. \& Kornman, K. S. Staging and grading of periodontitis: framework and proposal of a new classification and case definition. J. Periodontol, 89, S159-S172 (2018).

10.1002/JPER.18 - 0006

32. Trim, R. D., Skinner, M. A., Farone, M. B., Dubois, J. D. \& Newsome, A. L. Use of PCR to detect Entamoeba gingivalis in diseased gingival pockets and demonstrate its absence in healthy gingival sites. Parasitol. Res, 109, 857-864 (2011).

33. Wantland, W. W. \& Wantland, E. M. Incidence, ecology, and reproduction of oral protozoa. J. Dent. Res, 39 (863), https://doi.org/10.1177/00220345600390041801 (1960).

34. Wantland, W. W. \& Lauer, D. Correlation of some oral hygiene variables with age, sex, and incidence of oral protozoa. J. Dent. Res, 49, 293-297 (1970).

35. Weedall, G. D., Hall, N. \& Evolutionary genomics Entamoeba. Res. Microbiol. 162, 637-645(2011).

36. Yamamoto, A., Kikuta, N., Hashimoto, T., Oyaizu, H. \& Goto, N. Nucleotide sequence of the SrRNA gene of Entamoeba gingivalis: applications for construction of a species-specific DNA probe and phylogenetic analysis. Microbiol. Immunol, 39, 185-192 (1995).

\section{Figures}




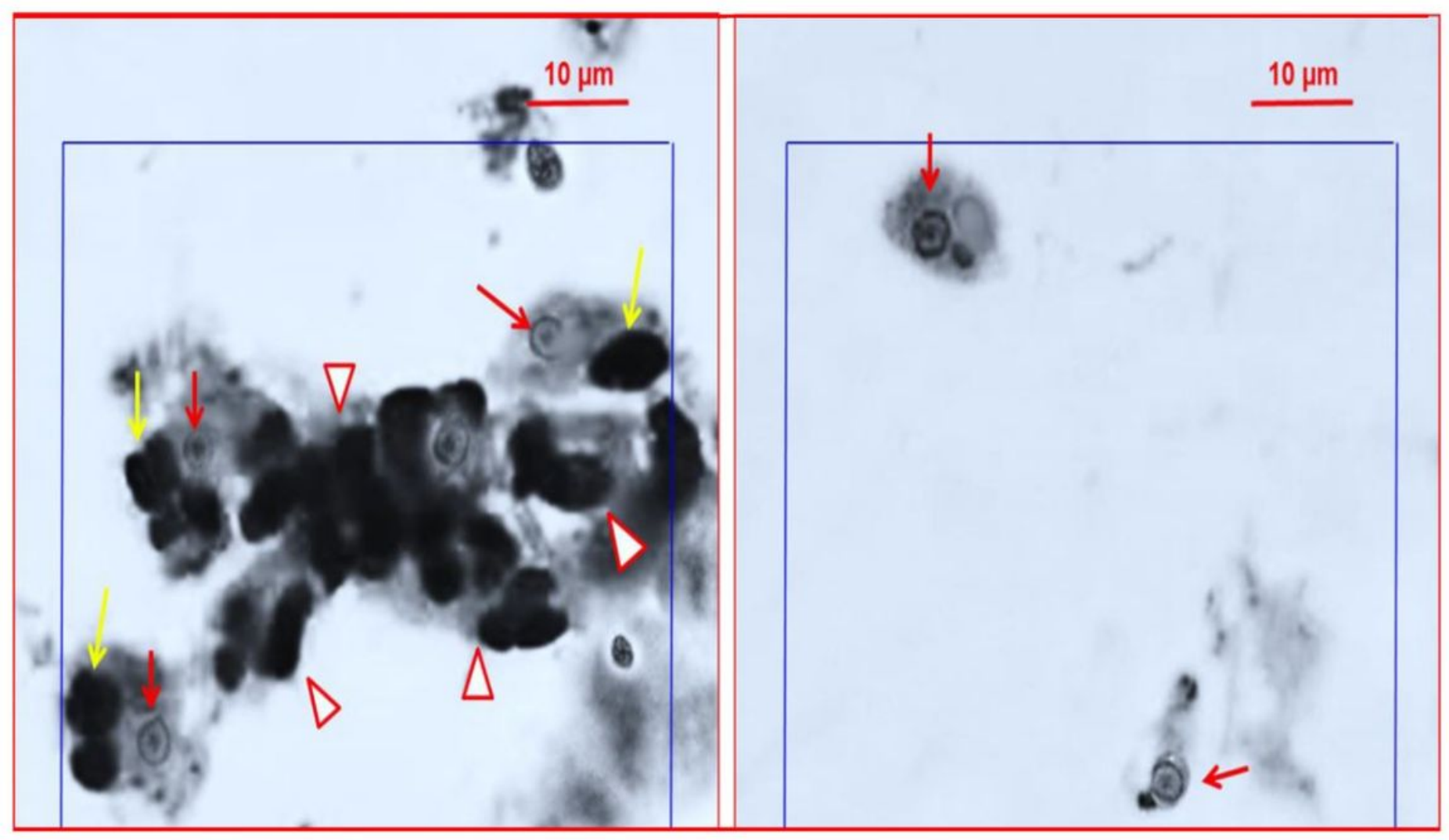

Figure 1

The morphological characteristic of Entamoeba gingivalis. A mass of Entamoeba gingivalis trophozoites stained with Iron and haematoxylin stain with characteristic nucleus (Red arrow) with extensive collection of cytoplasmic dark inclusion bodies, leuckophagocytic vacuoles (yellow arrows). Many trophozoites appear missing the characteristic nuclei (arrow heads). 

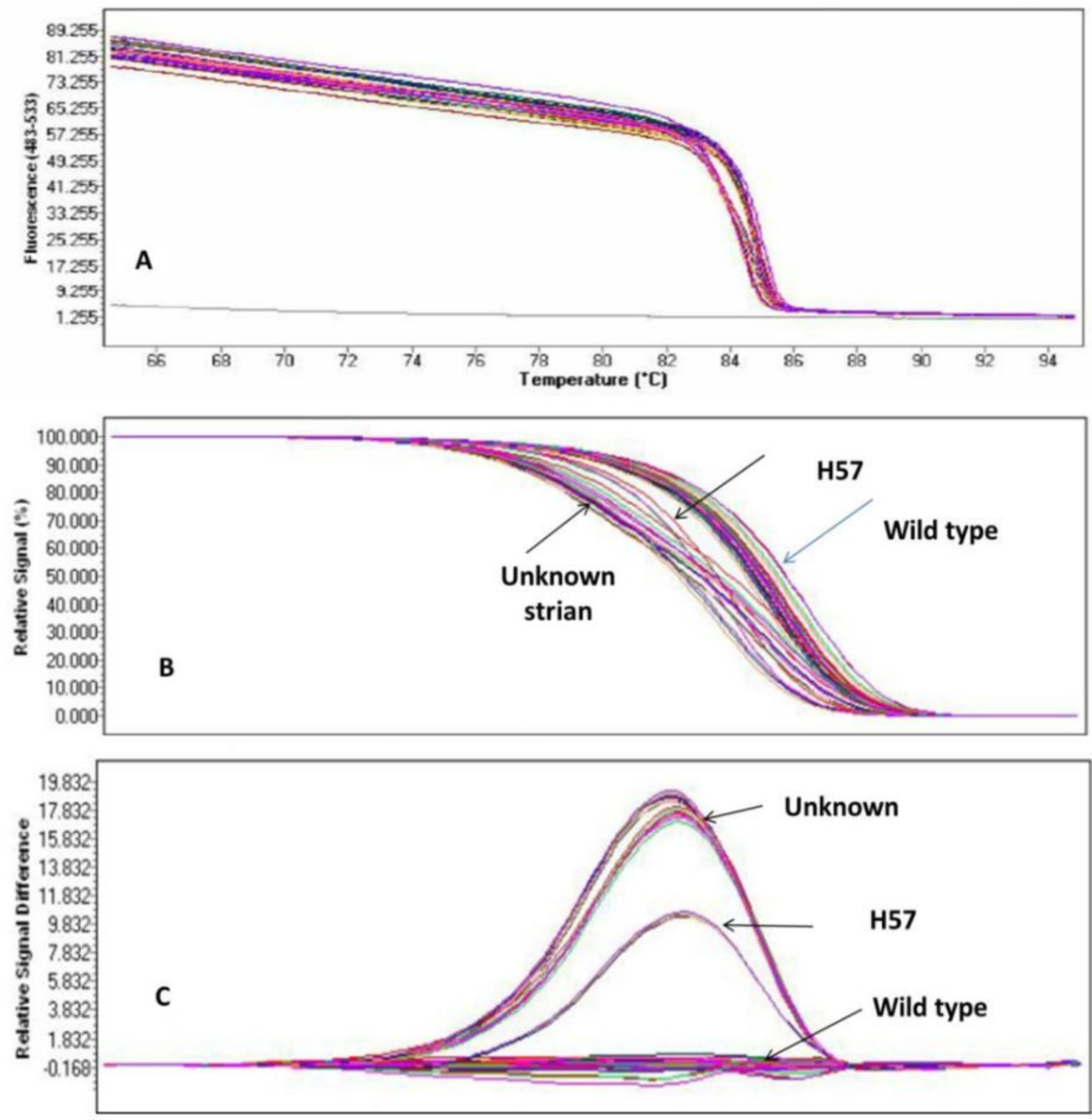

Figure 2

HRM analysis by real-time PCR. The results were generated by LightCycler 480 real-time PCR system and the mutation detection approaches A; pre-melt (initial) and post-melt (final) fluorescence signals of all samples. B; normalized melt curve to relative values. C; Curve differences as magnified by subtracting each curve from the most abundant type. 